\title{
Đại diện Trung tâm ISR thuyết trình tại seminar do VIASM tổ chức
}

\author{
Nguyễn Thanh Thanh Huyền
}

August 4, 2021

Phenikaa ISR (https:/_isr.phenikaa-uni.edu.vn/vi/_post/tin-tuc/tintuc/sub-tin-tuc/dai-dien-trung-tam-isr-thuyet-trinh-tai-seminar-doviasm-to-chuc) - Chiều ngày 4-8-2021 vừa qua, NCS. Nguyễn Minh Hoàng-Nghiên cứu viên Trung tâm Nghiên cứu Xã hội Liên ngành ISR, trường Đại học Phenikaa-đã có buổi thuyết trình tại Seminar Thống kê Úng dụng T8.2021 do Viện Nghiên cứu Cao cấp về Toán (VIASM) tổ chức.

Buổi thuyết trình "Bản hòa tấu dữ liệu xã hội” [1] được bắt đầu vào lúc 2 giờ chiều trên nền tảng Zoom do tình hình dịch bệnh căng thẳng trên cả nước. Tuy vậy, vẫn có khoảng 110 người tham gia buổi seminar. Trong số người tham dự, có nhiều giáo sư, phó giáo sư, giảng viên tới từ các trường đại học, viện nghiên cứu trên cả nước như Trường Đại học Kinh tế Quốc dân, Trường Đại học Kinh tế-VNU, Viện Khoa học Giáo dục Việt Nam, hay Tạp chí Giáo dục.

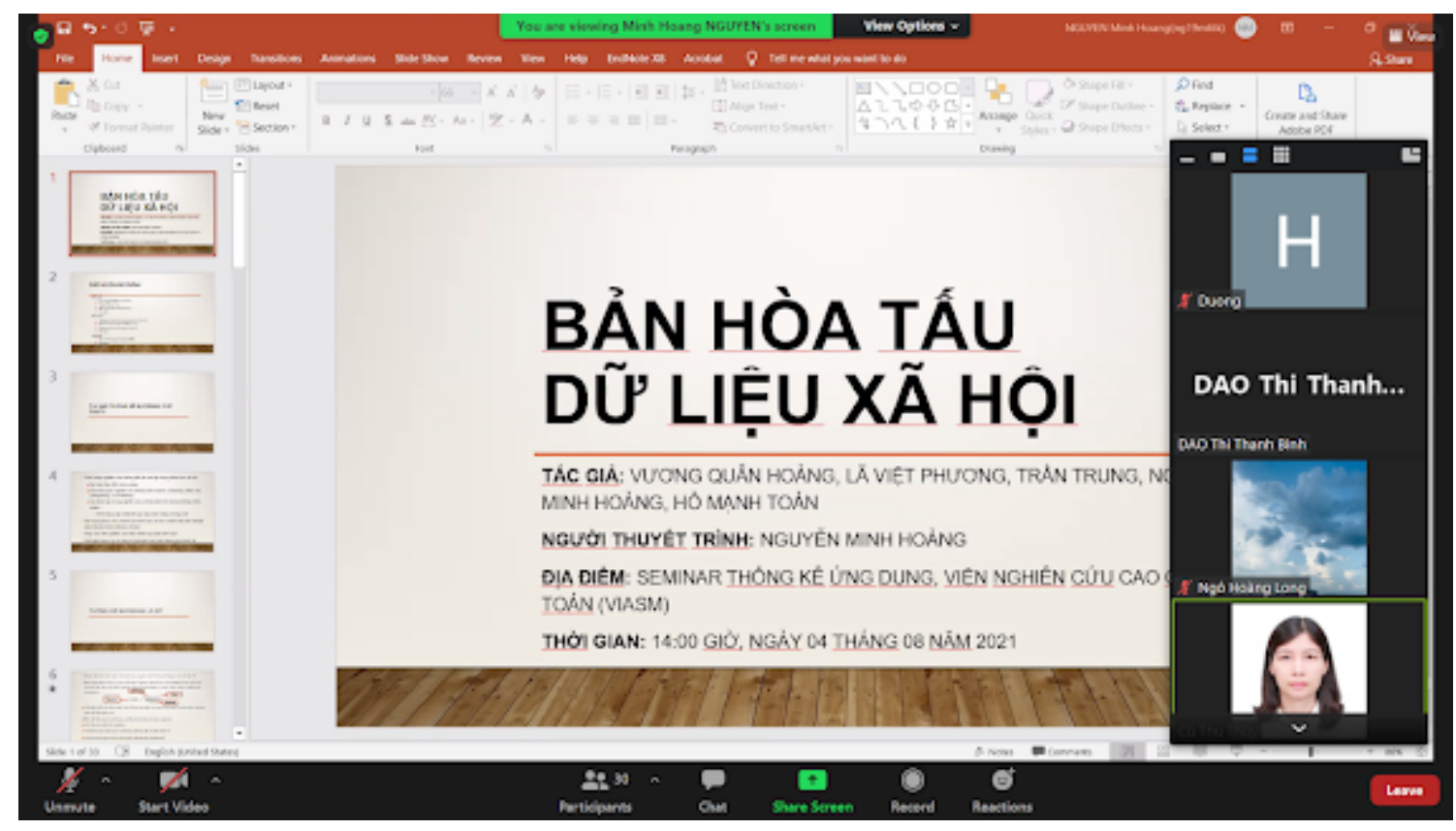

Buổi thuyết trình bắt đầu TS. Trần Trung (Học viện Dân tộc) giới thiệu về cuốn sách Bản hòa tấu dữ liệu xã hội, cảm hứng chính cho buổi seminar. Sau đó, trong hơn hai tiếng rưỡi đồng hồ, ThS. Nguyễn Minh Hoàng đã trình bày chuyên sâu giới thiệu thống kê Bayesian và chương trình bayesvl [2-3]. Phần trình bày bao gồm cả nội dung xử lý thuật toán, kiểm tra tính hợp lệ mô hình, và tìm hiểu ý nghĩa kết quả.

Trong lúc thảo luận, mọi người đã trao đổi sôi nổi về sự tương quan giữa thống kê Bayesian và thống kê truyền thống, cách ứng dụng bayesvl, cách đọc hiểu kết quả, cách trình bày, cách xây dựng mô hình, mã máy tính để 
chạy mô hình và có thể ứng dụng cụ thể cho lĩnh vực [4-6]. Nhiều người nghe cũng có đề nghị nên trao đồi về chủ đề này nhiều hơn trong tương lai.

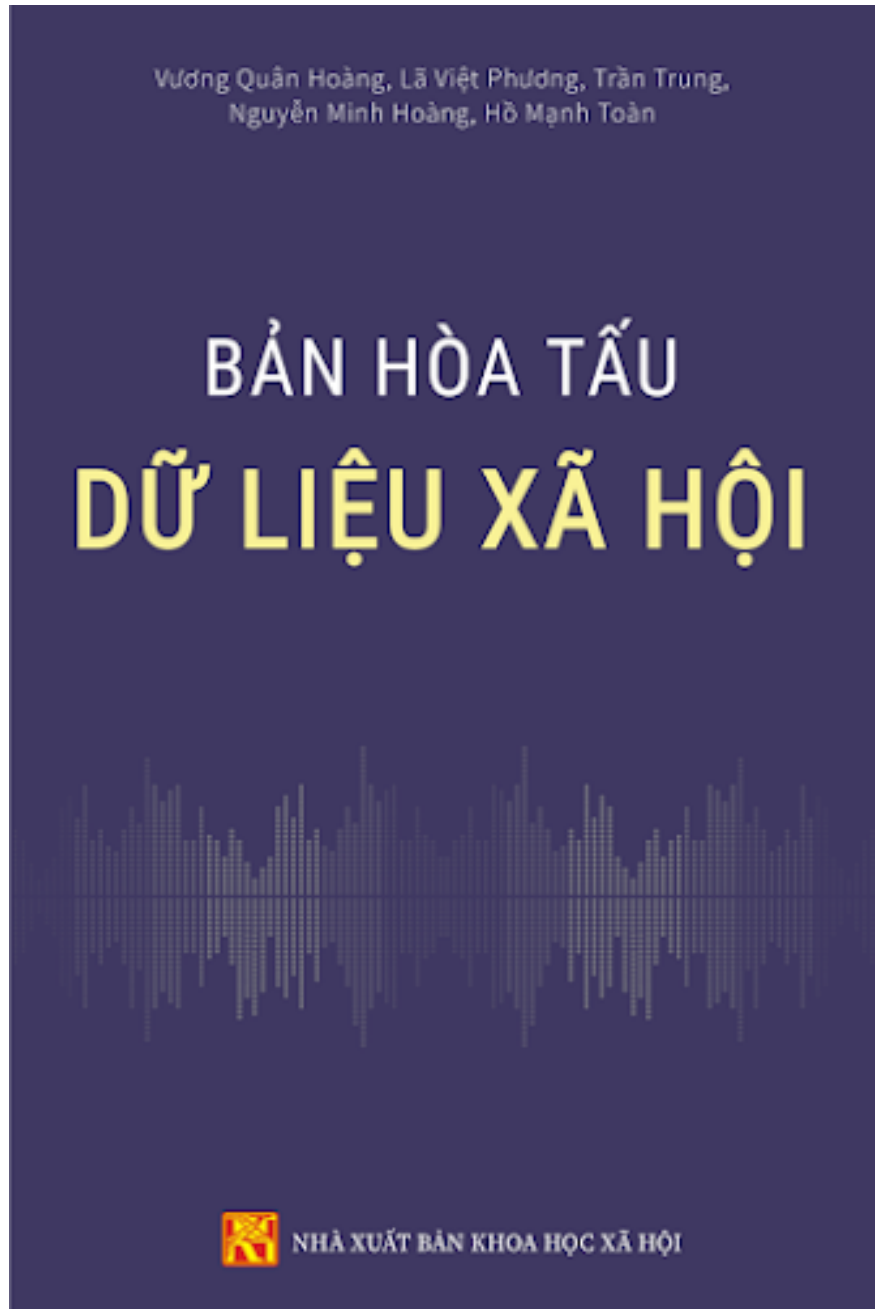

Seminar Thống kê Ứng dụng T8.2021 là chuỗi sinh hoạt chuyên đề hàng tháng của Viện Nghiên cứu Cao cấp về Toán (VIASM). Trong tháng 8 này, buổi sinh hoạt vào ngày 11-8 sẽ đón chào GS. Hồ Tú Bảo giới thiệu về học máy và khoa học dữ liệu. TS. Đặng Xuân Cương (Viện Khoa học Giáo dục) sẽ giới thiệu về lý thuyết phản ứng đáp câu hỏi trong hai buổi 18, 25 tháng 8. Thông tin có thể được tham khảo tại: https://viasm.edu.vn /hdkh/seminar-thong-ke-ung-dung-t8-2021

\section{Tài liệu tham khảo:}

[1] Hoàng VQ, Phương LV, Trung T, Hoàng NM, Toàn HM. (2021). Bản hòa tấu dĩ liệu xã hội. Nxb Khoa học Xã hội, Hà Nội. ISBN: 978-604-308-549-5. Available at: https://books.google.com/books /?id=eBU5EAAAQBAJ

[2] La VP, Vuong QH. (2019). bayesvl: Visually learning the graphical structure of Bayesian networks and performing MCMC with 'Stan'. The Comprehensive R Archive Network (CRAN). Available at:

https://cran.rstudio.com/web/packages/bayesvl/index.html

[3] Vuong QH, La VP. (2019). BayesVL package for Bayesian statistical analyses in R. github. Available at: https://github.com/sshpa/bayesvl 
[4] Vuong QH, La VP, Nguyen MH, Ho MT, Ho MT, Mantello P. (2020). Improving Bayesian statistics understanding in the age of Big Data with the bayesvl R package. Software Impacts, 4, 100016.

[5] Vuong QH, La VP, Nguyen MH, Ho MT, Tran T, Ho MT. (2020). Bayesian analysis for social data: A step-by-step protocol and interpretation. MethodsX, 7, 100924.

[6] Vuong QH, Ho TM, La VP. (2019). 'Stargazing' and p-hacking behaviours in social sciences: some insights from a developing country. European Science Editing, 45(2), 56-57. 